\title{
Development and Evaluation of Salbutamol Sulphate Loaded Ethyl Cellulose Microcapsules using Emulsion Solvent Evaporation Technique
}

\author{
Md. Shamsul Alam ${ }^{1}$, Jakir Ahmed Chowdhury ${ }^{2}$, Sams Mohammad Anowar Sadat ${ }^{2}$ \\ and Md. Selim Reza ${ }^{2}$ \\ ${ }^{1}$ Department of Pharmacy, The University of Asia Pacific, Dhanmondi, Dhaka-1209, Bangladesh \\ ${ }^{2}$ Department of Pharmaceutical Technology, University of Dhaka, Dhaka-1000, Bangladesh
}

Received: May 05, 2015; Accepted: June 14, 2015; Published (Web): July 21, 2015

\begin{abstract}
Ethyl cellulose (EC) microcapsules containing Salbutamol sulphate (SS) were prepared through emulsion-solvent evaporation technique. Microcapsules were compressed and in-vitro release profiles were studied from both microcapsules and their compressed matrix tablets. Different amounts of drug were added in order to obtain various drugs to polymer ratios and it was found that the size of microcapsules reduced with the increase in core loading. In the preparation of formulations, Tween 80 was used as an emulsifying or dispersing agent and light liquid paraffin (LLP) was used as oil phase. The in-vitro release of EC microcapsules was studied in distilled water at $37^{\circ} \pm 0.5^{\circ} \mathrm{C}$. A biphasic release behavior of SS from microcapsules was observed. In case of microcapsules, an immediate release was observed but for their compressed tablet form, initially a burst effect and then slow release were observed which was extended for 8 hours. In order to further investigate the type of drug release mechanism, the dissolution data were plotted according to the different kinetic models. In-vitro dissolution studies showed that zero-order and square-root of time (Higuchi model) release characteristics were exhibited.
\end{abstract}

Key words: Ethyl Cellulose, Salbutamol Sulphate, Microcapsule, Emulsion-Solvent Evaporation Technique.

\section{Introduction}

Salbutamol sulphate is a potent $\beta-2$ adrenoceptor stimulant which is used for the treatment of reversible airways obstruction. It is readily absorbed from the gastrointestinal tract when administered orally. Its biological half life is about 4 to 6 hours. In recent years, the number of patients with chronic diseases is increasing day by day. Taking a lot of medicines simultaneously, this can lead to reduced patient compliance. This problem is serious for such drugs with short biological half lives because they must be taken more frequently. In this situation, it is necessary to develop drugs for a longer period. One method to solve such problems is to develop a dosage form capable of releasing the drug gradually. In this regard, microencapsulation has been used as one of the tools to formulate controlled drug delivery systems (Yamada et al., 2001).

Ethyl cellulose (EC) is generally considered as a nontoxic, biocompatible and non-biodegradable polymer.
These characteristics are the reasons of its extensive selection for development of oral dosage forms, especially sustained release formulations. This is the reason why EC is extensively used for the development of oral multi-unit dosage forms (i.e. microcapsules). EC coated microcapsules have also demonstrated their capability to absorb pressure and therefore save the coating from fracture during tablet manufacturing process. This process involves the conversion of multi-unit system into a single unit dosage form by compression, which disintegrates slowly into sub-units when exposed to dissolution process (Rowe et al., 2003).

Alteration of any independent variable factor like emulsifier type, rate of organic solvent evaporation, core loading, rate of stirring, core solubility etc may affect the final microcapsule characteristics and release kinetics (Fong et al., 1986; Tice et al., 1985; Spenlehauer et al., 1986; Wakiyama et al., 1982 a,b; Jalil et al., 1990). The purposes of the present study were: a) to prepare and

Correspondence to: Jakir Ahmed Chowdhury; Tel: +880-2-7219747; Fax: +880-2-8615583; E-mail: jakirdu96@ gmail.com 
characterize SS microcapsules using EC as wall material by the emulsion-solvent evaporation technique; b) to determine the effect of the polymer to drug ratio on invitro dissolution study; c) to investigate how microcapsules and their compressed matrix tablet form affect the drug release; d) to fit the data to various postulated drug release kinetic models.

\section{Material and Methods}

Materials and reagents: Salbutamol sulphate (Incepta Pharmaceuticals Ltd.), Ethyl cellulose (Ethocel $20 \mathrm{cps}$, Colorcon, USA), Light liquid paraffin (MERCK, Germany), Tween 80 (MERCK, Germany), acetone (MERCK, Germany), n-hexane (MERCK, Germany) were used in this study from the indicated sources.

Preparation of ethyl cellulose microcapsule: EC microcapsules containing SS were prepared using the emulsification and organic solvent evaporation technique (Jalil et al., 1989). Acetone was used as polymer solvent, light liquid paraffin (LLP) as the microcapsulating vehicle and n-hexane as the decanter of paraffin oil. To prepare microcapsules with various drug to polymer ratios such as $1: 1,1: 2$ and $1: 3$, an amount of $1.8 \mathrm{~g}$ of polymer (EC) was weighed and either $1.8,0.9$ or $0.6 \mathrm{~g}$ of SS was added, respectively. The drug to polymer ratio was varied keeping the amount of polymer and solvent constant in all cases and decreasing the amount of drug used. An amount of $1.8 \mathrm{~g}$ of polymer (EC) was dissolved in $15 \mathrm{ml}$ of acetone. Depending on the desired ratio, weighed amounts of SS were dispersed in this solution and stirred for 20 min by a high speed stirrer (Heidolph, England). The dispersion was poured into $100 \mathrm{ml}$ of LLP containing 1.3 $\%$ Tween 80 and stirred for 3 hours at $1500 \mathrm{rpm}$ at room temperature. After 3 hours of stirring, the solvent for EC was completely evaporated and hard as well as spherical shaped microcapsules were found. Prepared microcapsules were then washed twice with $100 \mathrm{ml}$ of $\mathrm{n}$-hexane and consequently were separated by filtration. A vacuum dryer (VEEGO, India) was used to dry to obtain free-flowing microcapsules.

\section{Evaluation of microcapsules prepared with ethyl cellulose}

Drug content determination: At room temperature, stock solutions of SS (0 to $18 \mu \mathrm{g} / \mathrm{ml}$ ) in distilled water were prepared and the absorbance was measured by UVVIS Spectrophotometer (UV mini-1240, SHIMADZU CORP., Kyoto, Japan) at $276 \mathrm{~nm}$. A linear line was obtained while absorbance values were plotted against concentrations $\left(\mathrm{R}^{2}=0.9993\right)$.

Drug (SS) loaded EC microcapsules of each batch were finely powdered in a glass mortar. A clear solution of the powder was made using distilled water after proper sonication by a sonicator (POWER SONIC 505, HWASHIN TECHNOLOGY CO., Seoul, Korea). The solutions of the microcapsule samples were subjected to ultrasonication in order to achieve the complete breakage of the microcapsule wall and to release the total drug content. Therefore, the samples of solution were filtered through $0.45 \mu \mathrm{m}$ filter and analyzed spectrophotometrically for drug content (Gohel et al., 1998; Florey 1990).

The drug loading (\%) was calculated using the following equation:

$$
\text { Drug lcading }(\%)=\begin{gathered}
\text { Mmourt ef dug found in microcapsules } \\
\text { Amount of drug uscd for mi crocrcap oulatior. }
\end{gathered} 100
$$

The encapsulation efficiency (\%) was determined by the following equation:

$$
\text { Eucausulatior. entiency (\%) }-\frac{\text { Actual dizg logding }}{\text { Theorelical drug lozdiug }} \times 100
$$

In vitro dissolution study: Microcapsules of a particular size range were separated with the help of a sieve set (Endecotts Limited, England) for dissolution study. The USP paddle method was used to determine release of SS from the microcapsules which was carried out in a USP XXX apparatus Type-2 (Paddle Apparatus) in $900 \mathrm{ml}$ distilled water of $37^{\circ} \pm 0.5^{\circ} \mathrm{C}$ at a rotational speed of $50 \mathrm{rpm}$. Dissolution samples were withdrawn at 
appropriate intervals up to 8 hours and then filtered through $0.45 \mu \mathrm{m}$ filters. The drug content was determined spectrophotometrically at $\lambda_{\max }=276 \mathrm{~nm}$ in the filtrate either directly or after appropriate dilution with the dissolution media. The dissolution study for each batch was performed in triplicate.

Kinetic models: All data obtained from in-vitro release studies were fitted to various kinetic equations to find out the mechanism of drug release from the EC microcapsule. In this study, the kinetic models (1) zeroorder kinetics (Eq. 1) and (2) square-root of time equation (Eq. 2) used were:

Zero-order equation : $Q_{t}=k_{0} t \ldots \ldots \ldots \ldots 1$

Higuchi equation based on

Fickian diffusion : $Q_{t}=k_{H} \sqrt{ } t \ldots \ldots \ldots \ldots .2$

Where, $Q$ is the amount of drug release in time t, $k_{0}$, and $k_{H}$ are rate constant of zero order and Higuchi rate equations respectively. Determining the correlation coefficient assessed fitness of the data into various kinetic models. The rate constants, for respective models were also calculated from slopes.

\section{Results and Discussion}

Encapsulation efficiency: The drug to polymer ratio varied, maintaining the amount of polymer and solvent constant in all cases and decreasing the amount of drug used. As the drug to polymer ratio increased the microcapsules' mean size decreased (Table 1). The reduction in microcapsule size with increase in drug to polymer ratio may be due to a decrease in the viscosity of the internal phase as a result of a decrease in the concentration of solids in polymer solution.

In vitro release kinetics: The effect of variation in drug to polymer ratio on drug release was studied on SS microcapsules and their compressed matrix tablet forms in a dissolution medium of distilled water. The release profile of all microcapsule formulations was characterized by a typical initial burst effect due to SS release from the surface of the microcapsules. The rapid release attributed to leaching of drug through pores and channels after water imbibition.

Table 1. Physical properties of SS-EC microcapsules.

\begin{tabular}{ccccc}
\hline Formulations & $\begin{array}{c}\text { Drug/polymer } \\
\text { ratio }\end{array}$ & $\begin{array}{c}\text { Size: arithmetic diameter }(\mathrm{d}) \\
(\mu \mathrm{m})( \pm \text { S.D. })\end{array}$ & \multicolumn{2}{c}{ Loading efficiency $(\%)$} \\
\cline { 3 - 5 } & $1: 1$ & $946.21 \pm 356$ & 50 & Theoretical \\
\hline SM50 & $1: 2$ & $829.76 \pm 142$ & 33 & 46.52 \\
SM25 & $1: 3$ & $698.29 \pm 213$ & 25 & 31.41 \\
\hline
\end{tabular}

Table 2. Release rate parameters [y-equation $(Y=a X+b)$, correlation co-efficient $\left(R^{2}\right)$ and release rate] for release data after fitting of the whole release profiles of salbutamol sulphate.

\begin{tabular}{|c|c|c|c|c|c|c|c|}
\hline \multirow{3}{*}{$\begin{array}{l}\text { Formul- } \\
\text { ations }\end{array}$} & \multirow{3}{*}{$\begin{array}{l}\text { Drug / } \\
\text { polymer } \\
\text { ratio }\end{array}$} & \multicolumn{6}{|c|}{ Kinetic models } \\
\hline & & \multicolumn{3}{|c|}{ Zero-order } & \multicolumn{3}{|c|}{ Higuchi } \\
\hline & & Y-equation & $\mathrm{R}^{2}$ & $\begin{array}{c}\text { Release } \\
\text { rate }\end{array}$ & Y-equation & $\mathrm{R}^{2}$ & Release rate \\
\hline SM50 & $1: 1$ & $y=92.377 x+23.744$ & 0.7772 & 92.377 & $y=107.83 x+1.6245$ & 0.922 & 107.83 \\
\hline SM33 & $1: 2$ & $y=84.93 x+17.8$ & 0.8304 & 84.93 & $y=14.654 x+25.128$ & 0.6174 & 14.654 \\
\hline SM25 & $1: 3$ & $y=69.606 x+13.859$ & 0.8800 & 69.606 & $y=97.211 x-1.401$ & 0.9472 & 97.211 \\
\hline SMC50 & $1: 1$ & $y=3.9597 x+34.497$ & 0.4036 & 3.9597 & $y=15.071 x+21.217$ & 0.6959 & 15.071 \\
\hline SMC33 & $1: 2$ & $y=4.1753 x+30.588$ & 0.4782 & 4.1753 & $y=78.67 x-1.2878$ & 0.9787 & 78.67 \\
\hline SMC25 & $1: 3$ & $y=3.7801 x+28.093$ & 0.4802 & 3.7801 & $y=13.603 x+19.664$ & 0.6945 & 13.603 \\
\hline
\end{tabular}




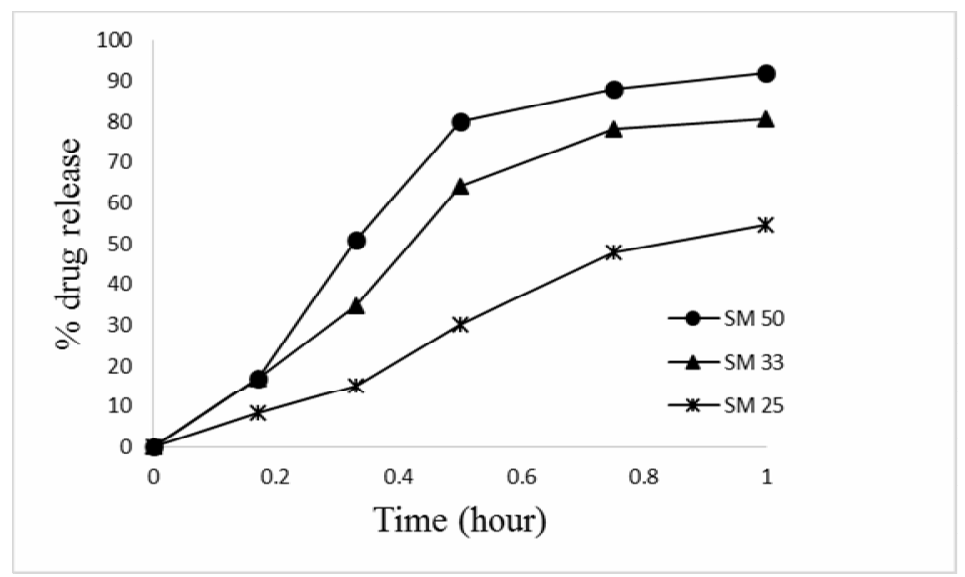

Figure 1. Zero-order plot of the salbutamol sulphate release from ethyl cellulose microcapsules prepared at different drug to polymer ratio as 1: 1 (SM50); 1:2 (SM33); 1:3 (SM25). Dissolution conditions: distilled water, temperature $37^{\circ} \pm 0.5{ }^{\circ} \mathrm{C}$, paddle rotation speed $50 \mathrm{rpm}$.

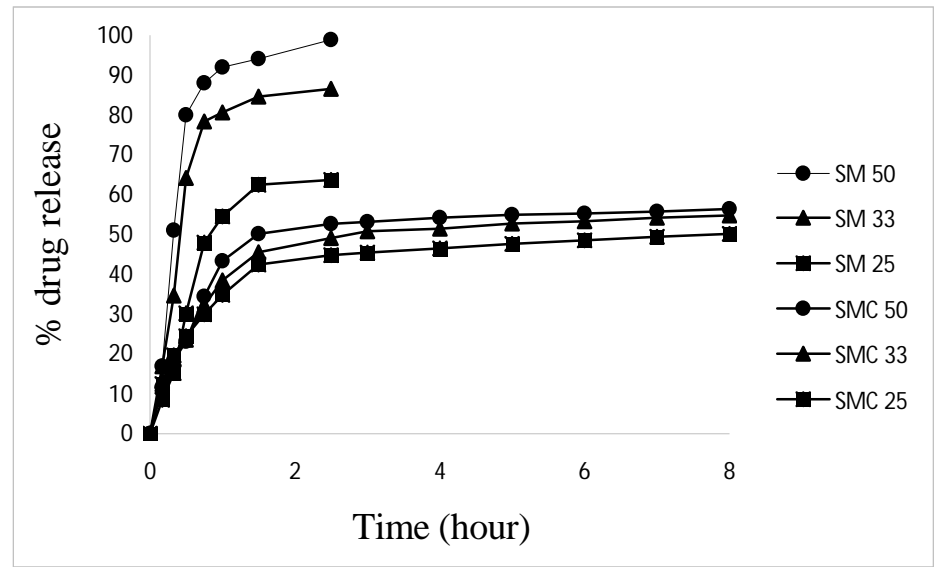

Figure 2. Zero-order plot of the salbutamol sulphate release from both ethyl cellulose microcapsules and their respective compressed matrix tablet forms prepared at different drug to polymer ratio (1:1), (1:2) and (1:3). Dissolution conditions: distilled water, temperature $37^{\circ} \pm 0.5^{\circ} \mathrm{C}$, paddle rotation speed $50 \mathrm{rpm}$.

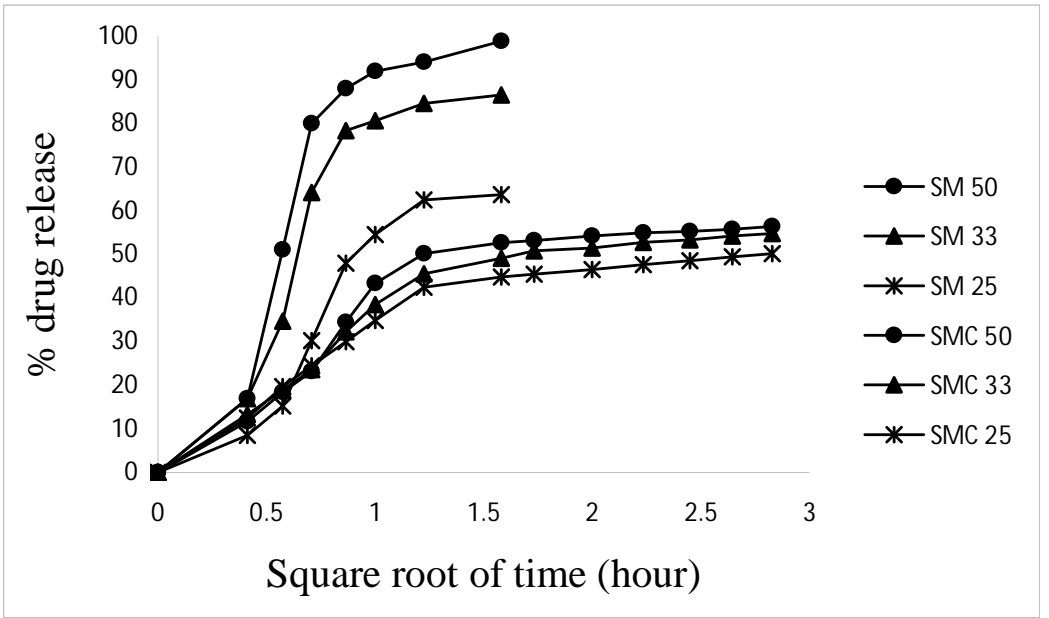

Figure 3. Higuchi plot of the salbutamol sulphate release from both ethyl cellulose microcapsules and their respective compressed matrix tablet forms prepared at different drug to polymer ratio (1:1), (1:2) and (1:3). Dissolution conditions: distilled water, temperature $37^{\circ} \pm 0.5^{\circ} \mathrm{C}$, paddle rotation speed $50 \mathrm{rpm}$. 
For the preparation of EC microcapsules containing SS, different drug to polymer ratio such as 1:1 (SM50), 1:2 (SM33) and 1:3 (SM25) were taken and found that increase in polymer concentration resulted in a decrease of drugs release rate (Figure 1). Initially, the compressed forms of the three respective formulations (SMC50, SMC33 and SMC25) showed burst release but after one and half hours a sustained release up to 8 hours was achieved. In case of compressed matrix tablet forms of the microcapsules, a decrease of drugs release was also found with increased in polymer concentration (Figure 2 and Figure 3). The releases of SS microcapsule were found to be higher as compared to their respective compressed matrix tablet forms' release. The release data obtained were fitted into two release kinetic models and release kinetics table (Table 2) was prepared.

\section{Conclusion}

EC Microcapsules containing SS were successfully prepared by W/O emulsion solvent-evaporation technique. It could be concluded that different drug to polymer ratio in the formulations influenced the variation observed in drug loading as well as entrapment efficiency, mean particle size and the drug release behavior. The results of this study may put forward the prospective application of ethyl cellulose microcapsules as a suitable sustainedrelease drug delivery system. Hence, a single unit sustained release oral dosage form of ethyl cellulose microcapsules containing salbutamol sulphate is promising to formulate twice daily dose for respective patients.

\section{References}

Florey, K. 1990. Analytical Profiles of Drug Substances, Academic Press Inc., New York. 19, p. 123.
Fong, J.W., Nazareno, J.P., Pearson, J. and Maulding, H.V. 1986. Evaluation of biodegradable microspheres prepared by a solvent evaporation process using sodium oleate as emulsifier. J. Control. Rel. 3, 119-130.

Gohel, M.C. and Amin, A.F. 1998. Formulation optimization of controlled release diclofenac sodium microspheres using factorial design. J. Control. Rel. 51, 115-122.

Jalil, R. and Nixon, J.R. 1990. Biodegradable poly(lactic acid) and poly(lactide-co-glycolide) microcapsules: problems associated with preparative techniques and release properties. J. Microencap. 7, 25-39.

Jalil, R. and Nixon, J.R., 1989. Microencapsulation using poly (L-Lactic Acid) I: microcapsule properties affected by the preparative technique. J. Microencap. 6, 473-484.

Rowe, R.C., Sheskey, R.J. and Weller, P.J. 2003. Handbook of Pharmaceutical Excipients. 4th ed. London: Royal Publishers, London, U.K., pp. 237-241.

Spenlehauer, G., Veillard, M. and Benoit, J.P. 1986. Formation and characterization of cisplatin loaded poly (d,l-lactide) microspheres for chemoembolization. J. Pharm. Sci. 75; 750-755.

Tice, T.R. and Gilley, R.M. 1985. Preparation of injectable controlled-release microcapsules by a solvent-evaporation process. J. Control. Rel. 2, 343-352.

Wakiyama, N., Juni, K. and Nakano, M. 1982a. Influence of physicochemical properties of polylactic acid on the characteristics and in vitro release patterns of polylactic acid microspheres containing local anesthetics. Chem. Pharm. Bull. 30, 2621-2628.

Wakiyama, N., Juni, K. and Nakano, M. 1982b. Preparation and evaluation in vitro and in vivo of polylactic acid microspheres containing dibucaine. Chem. Pharm. Bull. 30, 3719-3727.

Yamuda, T., Ohnishi, H. and Machida, Y. 2001. Sustained release ketoprofen microparticles with ethyl cellulose and carboxymethyl ethyl cellulose. J. Control. Rel. 75, 271 282. 